\title{
Transformation of education in the conditions of the information society development: institutional and cultural aspects
}

\author{
Marina Danilova*, Napoleon Plotnikov \\ Kuban State Agrarian University named after I.T. Trubilina, 350004, Krasnodar, Russia
}

\begin{abstract}
The article raises the question of changes affecting the modern education system in the context of the extensive sociocultural transformations that have occurred in recent decades. The main emphasis in considering the changing conditions for the implementation of educational activities is associated with the study of the development processes of the information society. The theoretical premise of the study is the idea of changing the functional balance of the educational sphere, associated with the need for its conformity with modern social challenges. In particular, the article analyzes the relationship between education and training in educational activities. The study also poses the problem of dynamic discrepancies between transformation processes in society and in the educational sphere. The essence of this discrepancy is that the achievements of science and culture with a significant delay become the property of educational activity, and the structure of higher educational institutions often does not meet the need for training specialists in a number of innovative areas. On this background, specialized courses and various network resources, focused on filling the "gaps" of the centralized education system, are becoming relevant. At the end of the article, a conclusion is drawn regarding the main trends in the transformation of education in modern society.
\end{abstract}

\section{Introduction}

*Corresponding author: madary@mail.ru 
The current stage of society development is considered to be unique. Despite the fact that the understanding of the current processes in culture and society is greatly facilitated by the understanding of the general principles and trends that have been noted in humanitarian knowledge for a long time, large-scale qualitative changes that have affected both the social sphere and the field of culture cannot be ignored. The main importance of qualitative changes in society for science is that fundamentally new trends, relationships, and relationships are formed. Large-scale shifts are taking place at the level of the cultural sphere, all the consequences of which have not yet been determined (Ponarina, 2012); the scope of the economy has significantly changed, which affects the distribution of jobs and the demand for various specialties; There is an intensive development of scientific and technical fields, accompanied by a tremendous increase in the general level of available knowledge.

Under these conditions, the development of appropriate, fundamentally new approaches to understanding the contemporary sociocultural sphere is required. At the same time, the issue of adapting the cultural sphere to change sociocultural conditions is especially acute (Cheshev, 2017). One way to solve this problem is to change the educational sphere since education is one of the main mechanisms of cultural inheritance (Danilova \& Sukhoverkhov, 2015). Characterizing at a general level the spectrum of problems that must be resolved within the educational sphere, the following problems should be separately distinguished:

- overcoming crisis trends in social culture;

- transfer during the educational process of relevant knowledge (rather than outdated concepts);

- achieving compliance between the demands of the labor market and the set of specialties that are being formed at the present stage;

- preservation of the educational function for the education system;

- maintaining the quality standards of educational activities in the context of the numerous alternatives development to modern universities.

The above range of problems is associated with two major trends - increasing the transformational dynamics in society and updating information processes associated with the development and popularization of the global Internet.

The relevance of the problems study for modern education is associated with the fundamental importance of the educational sphere for society, and at the same time, with the insufficient knowledge of the modern aspects for educational activity related to the unique qualitative characteristics of the social development modern stage. The object of the study is modern sociocultural factors of education; the subject is the influence of the development processes for the information society on the system of modern education. The purpose of this research is to determine the main specific factors and features of the educational function implementation at the modern historical stage. To implement this function, the following tasks are defined:

- give a general description of modern sociocultural changes;

- analyze the main functions of the educational sphere;

- identify the main range of modern education problems; 
- correlate the main functions and problems of the education system;

- identify the main trends and prospects for the development of the educational sphere in modern conditions.

The main methods used in this article include comparison, analysis, deduction, as well as historical and structural-functional approaches.

\section{Sociocultural context of modern education}

Two main criteria that characterize processes in the cultural environment and at the level of social structure as well as possible are dynamics and complexity. Over the past century, there have been a number of significant changes in society, which determined an extreme increase in the intensity of social processes and a significant complication of the social structure. Moreover, speaking of intensity, we mean both ordinary, cyclically repeating processes, and transformational dynamics. Society has become more complex, multi-component, and the frequency of functional contacts in it has increased significantly. Moreover, if previously there was an element of stability in the way of life (with the exception of serious social upheaval moments, for example, wars), then at present the speed of radical social changes is extremely high. Under such conditions, it is not only quite difficult for a person to navigate in public life, but it is also necessary to continuously adapt to its changes (Danilchuk, 2002).

The complexity and multiplicity of culture, the high dynamics of social changes in the aggregate lead to a violation of the public life stability. Differences between people are exacerbated, which becomes the basis for an increase in the level of conflict; at the same time, adherence to generally accepted moral standards is reduced due to the pluralism of value attitudes presented in the culture. All this actualizes the humanitarian component of the educational process, and at the same time significantly complicates the process of developing vocational training, since the high dynamics of social changes in the context of a cumbersome training system, which requires several years of preparation, can in some cases determine the irrelevance of the education received. This is due to both obsolescence of knowledge, methods, technologies, and changes in the labor market, in which the specialty loses its relevance for the period that students spend on vocational training. The following dilemma is acute: the universalism of a educational programs number contributes to the implementation of the education humanitarian function, which is associated with an increase in the awareness of public life and the inculcation of constructive values (and, in general, an increase in orientation ability of the social environment). However, the implementation of the universalism installation in education entails an increase in its terms. Thus, the society is interested in implementing a large-scale, multi-subject educational process, however, the individual members' interests of the society (primarily, conscious priorities are implied) are increasingly located in the field of fast, effective, profile-oriented education. According to the researchers, the conflict between the humanitarian and pragmatic aspects of cultural development is one of the central in modern society, and the general trend is not in favor of the humanitarian sphere (Leschinskaya, 2017). 
Innovative professions deserve special attention and are reflected at the level of a centralized education system: between the occurrence of a specific current direction of professional activity and its inclusion in the curriculum of universities, there is a time interval associated with the recognition of the relevance of a training particular direction, the resolution of bureaucratic difficulties, the development and approval of the curriculum, training quality standards, etc. It is the education system slowness in combination with the obvious need for the training mechanisms development of specific areas determines the creation of private and corporate courses, which serve as an alternative to centralized education system in leading universities. Partly, the problem of adapting the educational process to new subjects is solved within the framework of the Western model of education, oriented towards independent choice by students from a wide range of educational courses offered. But here, too, the moment of new training course approval is fraught with significant difficulties, which ultimately entails an increase in the interval between the social request development for a specific area of educational activity and its implementation in the university system.

\section{The functional aspect of educational activity and its dynamics}

In the previous section, we described the specifics of the current state of society and the main factors of the institutional plan that determine the problems and vectors of modern education development. For a deeper understanding of the current situation, it is necessary to assess its impact on the functional side of educational activity, and, in particular, the changes that occur in it. The initial step in this regard is a general description of the functional aspect of educational activity. Consider the main functions of education. These include:

- socio-cultural functions of education (creation and development of the students' worldview, increasing their cultural level, broadening their horizons, increasing the creative potential of society members, instilling constructive moral and ethical attitudes, socialization, etc.);

- socio-economic functions of education (training specialists in the main spectrum of demanded specialties, growth of scientific potential, inculcation of universal skills that contribute to the growth of labor efficiency, etc.);

- socio-political functions of education (development of socio-political worldview that is more resistant to negative socio-cultural influences, the implementation of an ideological function, inculcation of qualities that contribute to the growth of social stability).

On the whole, the first and third groups largely overlap with the above division into the humanitarian and professionally oriented components of educational activity. And it should be noted here that the humanitarian component of education is being updated to a significant degree for society, however, it is a subject to gradual crowding out as part of the growing practice of passing specialized, profile-oriented education. There is a contradiction: socially oriented attitudes to the organization of the educational process determine its multifaceted nature, while economic orientation sets the development vector associated with the 
development of narrowly focused training. In conditions when the organization of the educational process is centralized, regulated by political power, one can judge about the vast possibilities for maintaining the completeness of the education social functions. At the same time, the emergence of educational processes at the level of self-organization inevitably leads to the actualization of the economic vector of the education sector development, which is currently observed both at the level of corporate training practice and at the level of specialized educational courses, many of which are presented at the network space level.

The above trends indicate the serious importance of information processes in the transformation of education. Moreover, the role of the information sphere as a means of ensuring educational activity is also important, and, at the same time, the importance of society informatization, as a factor in shifting the set of relevant training areas in those areas that arose thanks to the rapid development of digital technologies. This determines the relevance of addressing the informatization problem of modern society as a factor in educational activity.

\section{The information space of the Internet as a factor in educational activities}

Despite the diversity of the education functions, the key and most obvious of them is the transfer of knowledge. And in this regard, the mechanisms' development for storing and transmitting information is a serious help for organizing the educational process, and in a number of aspects it is the basis for replacing education in specialized institutions with selfeducation. The automated systems' development has determined a serious simplification of the acquiring information process on interest issues to a person. For this reason, the value of expert knowledge, which university professors possess, is gradually being lost, since network materials also contain a lot of expert recommendations, analytical materials, etc. For today's youth, "learning something" and "making a search query" are almost synonymous in an internet browser. Thus, Internet communication is becoming one of the main ways of implementing cognitive activity (Danilova \& Nikitin, 2018). This creates tremendous opportunities for the implementation of the educational process - viewing videos of highquality lectures, remote consultations with specialists, access to numerous, including rare, publications (Shevko \& Turutina, 2014), and, at the same time, devalues personal knowledge that is balanced the presence of a gadget with Internet access.

It should be noted separately that the use of Internet resources is fraught with significant risks, in particular - the materials' quality and reliability published in various network resources is not guaranteed, various inaccuracies, errors and even deliberate falsification are possible. However, at this stage we are not interested in the reliability of the Internet, but in its functionality. The transfer of knowledge in its purest form on a specific issue of interest to users is extremely practical. At the same time, it is in this practical, dry transfer of knowledge that we observe a reduction in education towards learning. Consider this aspect in more detail in the analysis of the distance education phenomenon. 
Remote education is the next step in the development of digital technologies in educational activities. For a long time, the Internet, as a huge repository of information, was a powerful tool for self-education. At the same time, the Internet is, first and foremost, a means of communication, and therefore it is quite logical that many communicative practices "take root" in the network communication system. In particular, modern digital communication sets the prerequisites for transferring the education institute to the network space, which is determined by factors such as the ability to transfer lecture courses in video format, as well as remote communication between the teacher and students. The obvious advantages of distance education are that it allows many people, due to their personal schedule, work, place of residence, health, etc., who do not have access to classical university education, still get access to the educational process (Kinelev, 2011 ) At the same time, there are a number of features that characterize the problem status of distance education:

- there is no teaching staff as an environment of socialization;

- in a significantly weakened form, mechanisms for monitoring the quality of training are presented;

- the educational process for the most part is a point-like nature, being oriented towards the transfer of a specific field of knowledge or a specific skill.

Thus, out of the entire spectrum of significant educational functions, only one is effectively implemented in the course of remote educational activities - in fact, focused on learning. As an addition to the educational process, taking into account the humanitarian functions of education, this mechanism can be assessed as valuable and useful. At the same time, if we talk about crowding out classical university education with point-based knowledge acquisition on the Internet, then there is a serious sociocultural problem.

\section{The problem of flexibility and universality of education}

The main problem, considered in the context of raising the question of the educational process organization in modern conditions, is the choice between such characteristics of education as flexibility and universality. The network communication system, while not burdened by a number of institutional constraints, has a significant measure of flexibility. At the same time, education received by members of society independently through an appeal to the field of network communication is extremely rarely widespread, universal in nature. In turn, the traditional system of higher education has a wide range of social functions, however, to a large extent loses by the criterion of the adaptation dynamics to changing social conditions. This characterizes the high relevance of improving the adaptive dynamics of the university education system, and also draws attention to the difference between the fields of knowledge, expressed in the degree of susceptibility to innovative development. The more intensively a certain professional or scientific-technical sphere develops, the more it makes demands on the organization of the educational process and the dynamics of its adaptation to changing conditions. And, on the contrary, the more stable and priority the accumulated layer of knowledge is, the more optimal are the training methods that have been verified over the 
years. This defines a sharp difference between the traditional, characterized by relative stability, areas of educational activity and innovation. As a matter of fact, it is with respect to the latter that the tendencies noted above for traditional institutions replacement of educational activity by the mechanisms of network learning arising as a result of selforganization processes are most acute.

\section{Conclusion}

Summing up the analytical review, we note that the main reason for serious transformations in the educational sphere and increased requirements for it lies at the junction of transformations in the social structure, cultural shifts and, in fact, the development of information and communication technologies. The Internet as a factor of education will be extremely narrow and incomplete if we pay attention to the possibilities of network resources for the implementation of educational activities and the alternatives to traditional universities that arise in the network space. The development of the information society has determined the emergence of a social requests' number, some of which are related to new requirements for the level of specialists' professional development, others - to the level of personal development for society members. The situation is aggravated by the fact that the traditional educational system does not have the measure of flexibility that a self-organizing digital communication system demonstrates. This determines the future of a innovative areas' number related to the implementation of private training mechanisms.

Against this background, one of the most promising ways of considering the emerging processes is, on the one hand, the separation of general educational processes and university education, and on the other, the consideration of differences between innovative fields and relatively stable areas of professional and scientific activity, the methods of organizing the educational process in which should vary significantly between themselves. At the same time, shifting the emphasis of cultural and educational measures in education towards their implementation in the system of compulsory education is gaining relevance, the search for alternative ways to implement these functions in society, as well as the search for ways to increase the competitiveness of the traditional education system.

\section{References}

1. V. V. Cheshev, Technical Progress in the Cultural and Historical Context, Voprosy Filosofii, 12, 64-78 (2017).

2. M. I. Danilova, G.M. Nikitin, The Phenomenon of "Virtualization" of the World in Modern Society, Astra Salvensis, 12, 661-663 (2018).

3. V.V. Leschinskaya, Technological Progress and the Problem of Humanization of Society in the Context of the Formation of Ecological Culture, Voprosy Filosofii, 10, 199-205 (2017). 
4. M.I. Danilova, A.V. Sukhoverkhov, Biological and social foundations of the evolution of language and communication: modern discussions, Questions of Philosophy, 12, 7787 (2015).

5. E.V. Danilchuk, Modernization of education in the information society and the information culture of a teacher, News of the Russian State Pedagogical University A.I. Herzen, 4, 153-161 (2002).

6. V.G. Kinelev, The philosophy of education in the XXI century. And its informational aspects, Bulletin of ChGAKI, 1 (25), 31-34 (2011).

7. N.N. Ponarina, Globalization and the Information Society, Society: Politics, Economics, Law, 1, 19-24 (2012).

8. N.R. Shevko, E.E. Turutina, Electronic educational resources as a tool for the development of educational space, Scientific notes KGAVM them. N.E. Bauman, 2, 324328 (2014). 